\title{
CAPACITANCE EVALUATION ON PERPENDICULAR PLATE CAPACITORS BY MEANS OF FINITE ELEMENT ANALYSIS
}

\author{
Jose-M. Bueno-Barrachina ${ }^{1}$, César-S. Cañas-Peñuelas ${ }^{1}$, S. Catalan-Izquierdo ${ }^{1}$, Francisco Cavallé-Sesé ${ }^{1}$ \\ ${ }^{1}$ Institute of Electrical Technology \\ 24, Av. Juan de la Cierva, Parque Tecnológico de Valencia \\ 46980, Paterna, Valencia (Spain) \\ Phone number:+0034 961 366670; Fax number:+0034 961366680 \\ e-mail: jmanuel.bueno@ite.es; cesar.canas@ite.es; saturnino.catalan@ite.es; fcavalle@ite.upv.es
}

\begin{abstract}
.
In this work we show the influence of the edge-effect on the electric field distribution, and hence on inner capacitance and outer capacitance of the inclined angle, of a inclined-plate capacitor system in a plane, surrounded by an insulating medium taking into account the thickness of the conducting plates for a complete set of dimensions and insulating characteristics. Where available we compare our results with previous published works. Finally, using statistical tools, we obtain the expression for computing the relationship between capacitance and insulation characteristics, insulation gap, plate dimensions and inclined angle.
\end{abstract}

\section{Key words}

Modeling, Electric Field, Capacitance, Finite Elements Method, Edge-effect.

\section{Introduction}

In the last years the development of the hybrid microelectronics technology [1], has opened new possibilities for the resolution of the technological problems that arise in every day from the Electrical Engineering. The development of etched fuses is of particular interest in the last three decades [2;3]. One of the advantages of this technology is the compression of the electric circuits. Nevertheless, the low distance between the conductors and the high potentials that they are created during the performance of a fuse causes that the capacitance between conductors and the electrical field between them is an important aspect to consider.

The aim of this work is to study the distribution of the electric field and the capacitance of an electric line constituted by two conductors of rectangular section and of unitary length. For this purpose, a few equations that allow to know about approximately from the capacitance of the capacitor generated in the applications where twoplate inclined plane capacitor of rectangular section was formulated. This inclination is due to the process of manufacture.

Nowadays, thanks to the performance that offer the actual computers, different iterative algorithms are used to solve the equations that govern the behaviour of this type of capacitors [4-8], these algorithms are designed in order to obtain the superficial charge distribution, the electric field values and the capacitance. The disadvantage of these algorithms is that they are designed for a few specific cases, so that if the geometry changes, the algorithm should be reconstructed completely.

Thanks to the development of the finite elements method (FEM), commercial software exist that integrate these method to solve the electrostatics generic problems, with independence of the geometry. This increase the flexibility and minimize the costs and the times of development. This is due to the fact that in these commercial software the graphic interface is employed to draw the geometry to analyze. When the geometry is drawn, the suitable boundary conditions are applied, which are necessary for a correct solution.

In this work, the FEM was implemented by the commercial software ANSYS [9], which has a specific module to solve electrostatics problems.

\section{Inclined plate capacitor}

The most common electrical engineering capacitor, is the one formed by two conductors.

Due to manufacturing and application constraints, one conductor plate is often inclined and not parallel to the other [10-12]. Recently, mathematical expressions based on hyperbolic functions were presented to calculate the theoretical capacitance of this type of capacitors, bearing in mind the " edge-effect". 


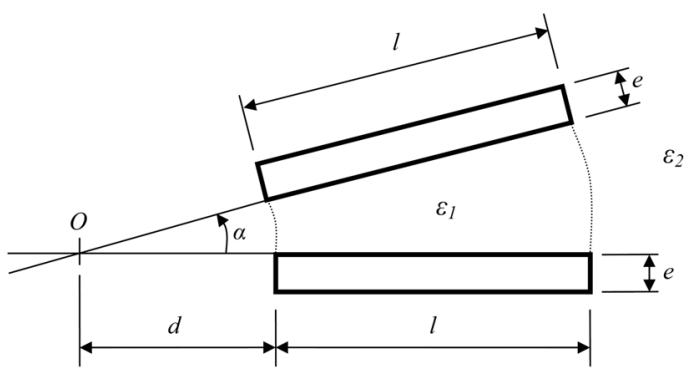

Fig. 1: Two-dimensional model of inclined capacitor.

In order to compare a real capacitor with one ideal one, a commercial software has been used to calculate the capacitance [10] and the electric field distribution between the conductors [13]. For this purpose, two-plane inclined plate capacitor has been generate, in which all relative permittivities and the geometric variables levels can be changed.

The geometry used in this model is shown in Fig. 1, where e it is the conductors thickness, $l$ is the conductors breadth, $d$ is the distance of the conductors to the coordinates origin, $\alpha$ is the inclination angle, $\varepsilon_{l}$ is the relative permittivity of the dielectric volume between the conductors and $\varepsilon_{2}$ is the relative permittivity of the dielectric volume that surround the system.

Table I shows the variables of the model with its corresponding values used for the simulation. Merging the values of this table, 3125 simulations have been carried out.

Table II show the results of the model simulation with an angle $\alpha=15^{\circ}$, a length $l=0.5 \mathrm{~m}$, a thickness $e=0.01 \mathrm{~m}$ and $\varepsilon_{2}=1$.

To evaluate the capacitance of the ideal capacitor, the equation proposed by Xiang, Y. can be used [11]. In this work the following mathematical exposition was employed [14].

Departing from the assumption of which both plates are in electrostatic balance, loaded with a constant charge $q$ uniformly distributed by the surface of the plates connected to a d.o.p. $\Delta V$.

As the electric field $\vec{E}$ is normal to the plates of the capacitor, the field lines alike to arcs of circumference (see Fig. 2) whose axis is the point $O$ (see Fig. 1). The electric field intensity has constant module along a field line and does not depend any more than of the radio $r$ of this one. So the potential difference $\Delta V$ between the armors is equal to the circulation of the field along a field line:

$$
\Delta V=\int_{L} \vec{E} \cdot \vec{d} l=\int_{\theta=0}^{\theta=\alpha} E(r) \cdot r \cdot d \theta=E(r) \cdot r \cdot \alpha
$$

The superficial charge density $\sigma$ on the armors, evaluated at a distance $r$ of the point $O$, is:

$$
\sigma(r)=\varepsilon_{0} \cdot \varepsilon_{1} \cdot E(r)
$$

From the equations (1) and (2) it obtains:

$$
\sigma(r)=\frac{\varepsilon_{0} \cdot \varepsilon_{1}}{r \cdot \alpha} \cdot \Delta V
$$

Table I: Variables of the model with its corresponding values used for the simulation.

\begin{tabular}{|c|c|c|c|c|c|}
\hline$\alpha\left(^{o}\right)$ & $l(m)$ & $e(m)$ & $d(m)$ & $\varepsilon_{1}$ & $\varepsilon_{2}$ \\
\hline 15 & 0.5 & 0.01 & 0.1 & 1 & 1 \\
\hline 30 & 0.875 & 0.0325 & 0.325 & 251 & 1 \\
\hline 45 & 1.25 & 0.055 & 0.55 & 500 & 1 \\
\hline 60 & 1.62 & 0.0775 & 0.775 & 750 & 1 \\
\hline 75 & 2 & 0.1 & 1 & 1000 & 1 \\
\hline
\end{tabular}

\begin{tabular}{|c|c|c|c|}
\hline$l / d$ & $\varepsilon_{1}$ & $C_{I D E A L}$ & $C_{\text {SIMULATION }}$ \\
\hline \multirow{5}{*}{5.000} & 1 & $6.06 \mathrm{E}-11$ & $1.51 \mathrm{E}-10$ \\
\hline & 251 & $1.11 \mathrm{E}-08$ & $1.39 \mathrm{E}-08$ \\
\hline & 500 & $2.22 \mathrm{E}-08$ & $2.77 \mathrm{E}-08$ \\
\hline & 750 & $3.32 \mathrm{E}-08$ & $4.15 \mathrm{E}-08$ \\
\hline & 1000 & $4.43 \mathrm{E}-08$ & $5.53 \mathrm{E}-08$ \\
\hline \multirow{5}{*}{1.540} & 1 & $1.36 \mathrm{E}-11$ & $2.28 \mathrm{E}-11$ \\
\hline & 251 & $3.42 \mathrm{E}-09$ & $5.73 \mathrm{E}-09$ \\
\hline & 500 & $6.82 \mathrm{E}-09$ & $1.14 \mathrm{E}-08$ \\
\hline & 750 & $1.02 \mathrm{E}-08$ & $1.71 \mathrm{E}-08$ \\
\hline & 1000 & $1.36 \mathrm{E}-08$ & $2.28 \mathrm{E}-08$ \\
\hline \multirow{5}{*}{0.909} & 1 & $8.05 \mathrm{E}-12$ & $1.65 \mathrm{E}-11$ \\
\hline & 251 & $2.02 \mathrm{E}-09$ & 4.15E-09 \\
\hline & 500 & $4.03 \mathrm{E}-09$ & $8.26 \mathrm{E}-09$ \\
\hline & 750 & $6.04 \mathrm{E}-09$ & $1.24 \mathrm{E}-08$ \\
\hline & 1000 & $8.05 \mathrm{E}-09$ & $1.65 \mathrm{E}-08$ \\
\hline \multirow{5}{*}{0.645} & 1 & $5.71 \mathrm{E}-12$ & $1.38 \mathrm{E}-11$ \\
\hline & 251 & $1.43 \mathrm{E}-09$ & $3.45 \mathrm{E}-09$ \\
\hline & 500 & $2.86 \mathrm{E}-09$ & $6.88 \mathrm{E}-09$ \\
\hline & 750 & 4.29E-09 & $1.03 \mathrm{E}-08$ \\
\hline & 1000 & $5.71 \mathrm{E}-09$ & $1.38 \mathrm{E}-08$ \\
\hline \multirow{5}{*}{0.500} & 1 & $4.43 \mathrm{E}-12$ & $1.22 \mathrm{E}-11$ \\
\hline & 251 & $1.11 \mathrm{E}-09$ & $3.06 \mathrm{E}-09$ \\
\hline & 500 & $2.22 \mathrm{E}-09$ & 6.09E-09 \\
\hline & 750 & 3.32E-09 & $9.13 \mathrm{E}-09$ \\
\hline & 1000 & $4.43 \mathrm{E}-09$ & $1.22 \mathrm{E}-08$ \\
\hline
\end{tabular}

Table II: Simulation results with an angle $\alpha=15^{\circ}$, a length $l=0.5 \mathrm{~m}$, a thickness $e=0.01 \mathrm{~m}$ and $\varepsilon_{2}=1$.

Taking one $d q$ of the surface of an armor, being:

$$
d q=\sigma(r) \cdot d S=\sigma(r) \cdot 1 \cdot d r
$$

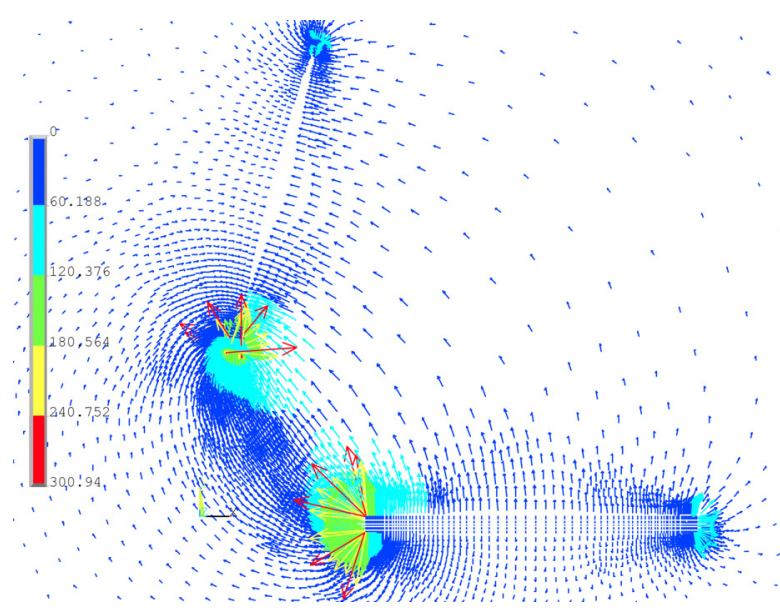

Fig. 2: Electric Field distribution on inclined plate capacitor with $\alpha=75^{\circ}, \mathrm{d}=1 \mathrm{~m}, \mathrm{e}=0.1 \mathrm{~m}, \mathrm{l}=2 \mathrm{~m}, \varepsilon_{1}=1000, \varepsilon_{2}=1$. 
Integrating $d q$ it is obtained:

$$
q=\frac{\varepsilon_{0} \cdot \varepsilon_{1}}{\alpha} \cdot \Delta V \cdot \int_{d}^{d+l} \frac{d r}{r}=\frac{\varepsilon_{0} \cdot \varepsilon_{1}}{\alpha} \cdot \Delta V \cdot \ln \left[1+\frac{l}{d}\right]
$$

Applying the capacitor general expression $q=C \cdot \Delta V$, the calculation of the capacitance is immediate.

$$
C=\frac{\varepsilon_{0} \cdot \varepsilon_{1}}{\alpha} \cdot \ln \left[1+\frac{l}{d}\right]
$$

Applying the Taylor series transformation to the logarithmic term of the equation (6), the following expression is obtained:

$$
C=\frac{\varepsilon_{0} \cdot \varepsilon_{1}}{\alpha} \cdot \sum_{n=1}^{m}(-1)^{n-1} \cdot \frac{(l / d)^{n}}{n}
$$

Fig. 3 shows graphically the values shown in the Table II. It can be seen in this figure that as it diminishes the ratio length - distance, there is more difference between the behaviour of a real capacitor and the ideal one. This difference is due to the fact that, as the plates of a capacitor approach, the distance with regard to the origin diminishes and the influence of the edge-effect in the capacitance also increases.

If we consider the plates charged with a constant charge $\mathrm{q}$, a secondary result of the simulation shows that the charge density at the top plate edge increase as it moves away from the low plate.

From the obtained results, the value of capacitance of a real capacitor according to 6 basic parameters was calculated; these are: angle of inclination $(\alpha)$, length $(l)$, separation $(d)$, thickness $(e)$, dielectric relative permittivity of dielectric volume between the plates of

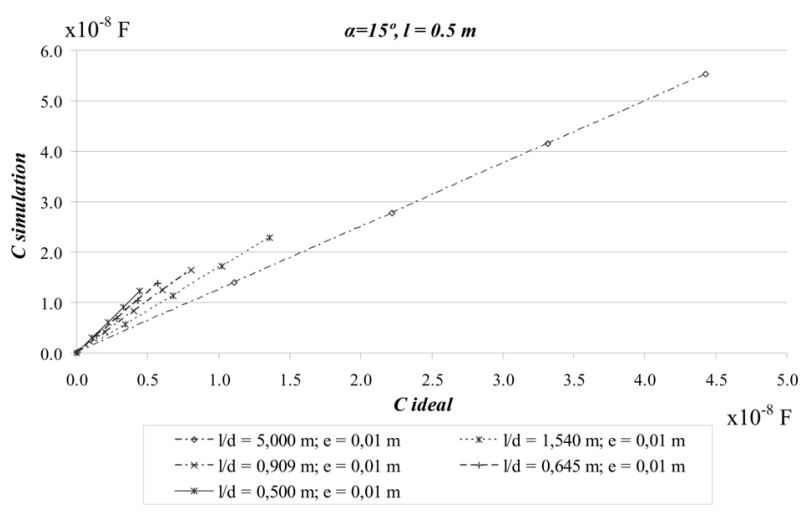

Fig. 3: Results of simulation, varying inter plates relative permittivity and separation.

the capacitor $\left(\varepsilon_{l}\right)$, dielectric relative permittivity of the dielectric volume that surround the plates $\left(\varepsilon_{2}\right)$.

By means of the employment of $\mathrm{n}$-dimensional technical analyses [15], the following polynomical function has been obtained that defines the behaviour of a real capacitor, for the particular case of which the dielectric volume between plates is not air.

The obtained $\mathrm{R}^{2}$ error in the interpolation is 0.9996 , which indicates that the equation fits well to the interpolated points.

\section{Model validation}

The validation of the model has been conducted comparing the values of the capacitance obtained in the simulation, with those calculated using the equation (10) proposed in [12], which is valid in the assumption of that the relative permittivity of the dielectric volume between the plates is vacuum, that is to say, $\varepsilon_{l}=1$, the lengths of the plates are equal, that reject the edge-effect and the thickness of the conductors. The $k_{\mathrm{R}}$ and $k_{\mathrm{L}}$ coefficients that appear in (11), are valid for the case of the perpendicular electrode plates (see Fig 1).

$$
C_{\text {regression }}=C_{\text {ideal }} \cdot A \cdot e+B \cdot d+C+D \cdot \alpha+E \cdot \frac{l}{d}+F \cdot l+G \cdot \varepsilon_{0} \cdot \varepsilon_{1}+H \cdot \varepsilon_{0} \cdot \varepsilon_{2}
$$

Where, the obtained coefficients are:

$$
A=1.2154 ; B=0.2184 ; C=2.2061 ; D=0.0077 ; E=0.0018 ; F=-0.1124 ; G=1.5127 ; H=-0.2799 \text {; }
$$

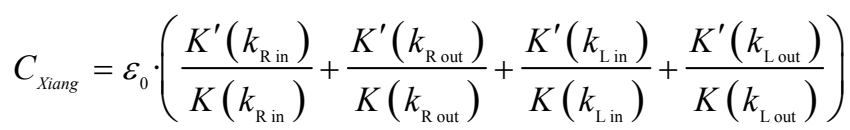

Where the coefficients $k_{\mathrm{R}}$ and $k_{\mathrm{L}}$ are calculated by the following expressions:

$$
\begin{array}{ll}
k_{\mathrm{R} \mathrm{in}}=\frac{r_{1}}{r_{1}+l_{1}} \cdot \sqrt{\frac{\left(r_{1}+l_{1}\right)^{2}+l_{2 \mathrm{R}}^{2}}{r_{1}^{2}+l_{2 \mathrm{R}}^{2}} ;} & k_{\mathrm{R} \mathrm{out}}=\sqrt{\frac{r_{1}^{(2 / 3)} \cdot\left(\left(r_{1}+l_{1}\right)^{(2 / 3)}+l_{2 \mathrm{R}}^{(2 / 3)}\right)}{\left(r_{1}+l_{1}\right)^{(2 / 3)} \cdot\left(r_{1}^{(2 / 3)}+l_{2 \mathrm{R}}^{(2 / 3)}\right)} ;} \\
k_{\mathrm{L} \mathrm{in}}=\frac{r_{1}}{r_{1}+l_{1}} \cdot \sqrt{\frac{\left(r_{1}+l_{1}\right)^{2}+l_{2 \mathrm{~L}}^{2}}{r_{1}^{2}+l_{2 \mathrm{~L}}^{2}}} ; & k_{\mathrm{L} \mathrm{out}}=\sqrt{\frac{r_{1}^{(2 / 3)} \cdot\left(\left(r_{1}+l_{1}\right)^{(2 / 3)}+l_{2 \mathrm{~L}}^{(2 / 3)}\right)}{\left(r_{1}+l_{1}\right)^{(2 / 3)} \cdot\left(r_{1}^{(2 / 3)}+l_{2 \mathrm{~L}}^{(2 / 3)}\right)}} ;
\end{array}
$$




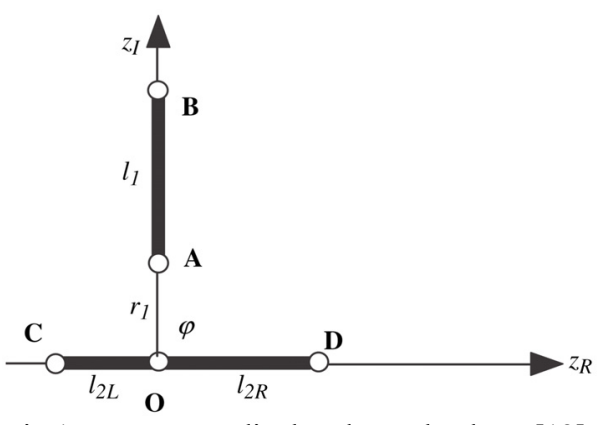

Fig 1. Two perpendicular electrode plates [12].

Fig 2 shows the calculated capacitance based in FEM with the assumption of Xiang, it can be seen that it fits to the capacitance calculated by Xiang method in [12], using the expression (10).

Fig 3 shows the electric field distribution in the model with the simplifications of the Xiang equations.

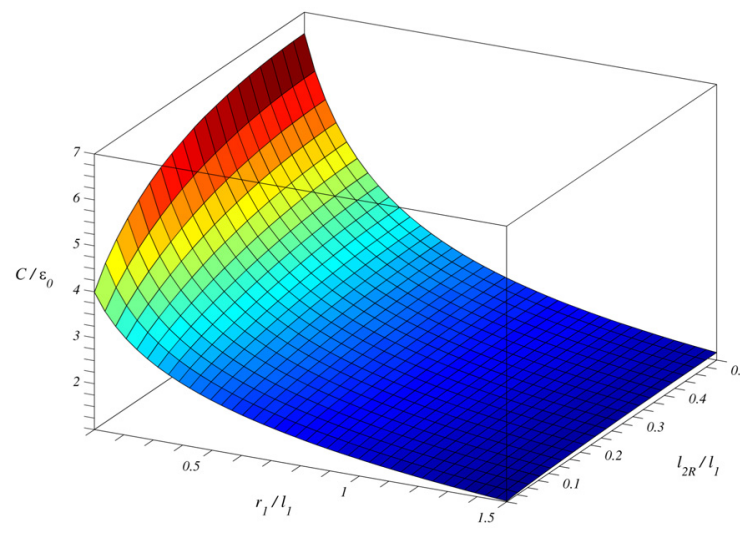

Fig 2. Simulation capacitances with Y. Xiang assumptions.
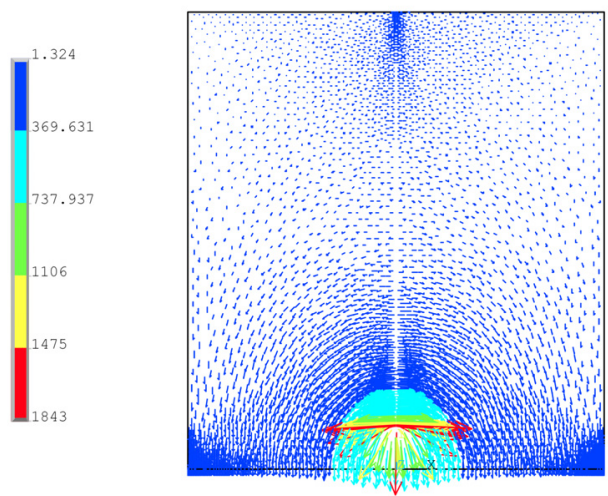

Fig 3. Electric field distribution with Y. Xiang assumptions.

\section{Conclusions}

In this paper it has been demonstrated the validity of FEM to calculate the capacitance between two-plates capacitor. For a certain angle it has been calculated the capacitance between two-plane inclined plate capacitor and a polynomical expression has been obtained, that allows to calculate the existing capacitance between two- plane inclined plate of rectangular section, considering the thickness of these.

This model also includes the volume that surrounds the conductors and considers the edge-effect of these.

In Fig 4 there appears the electric field distribution considering the edge-effect of the conductors and the volume that surrounds these.

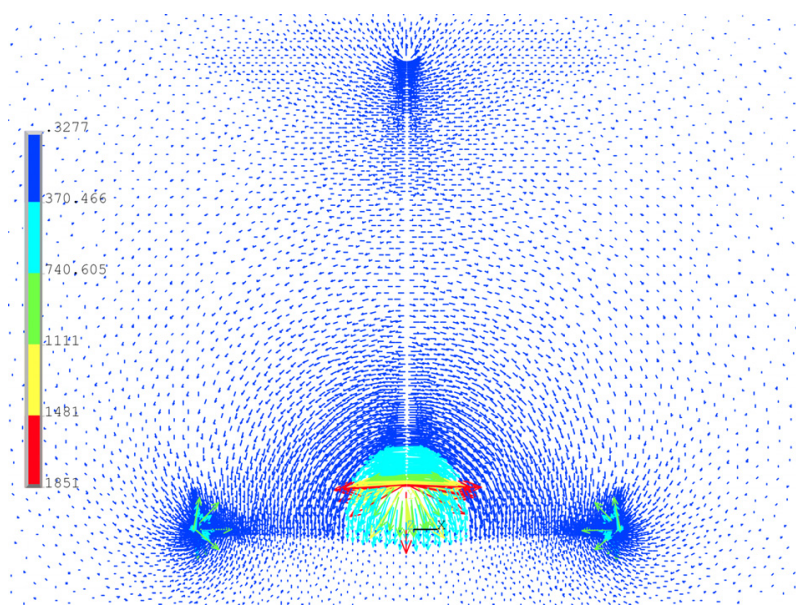

Fig 4. Electric field distribution.

Fig 5 shows the relation between the capacitance and the geometric model variables.

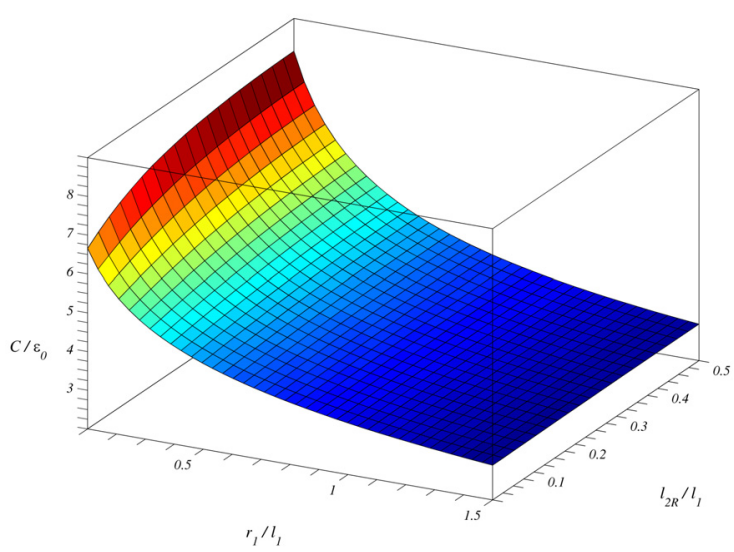

Fig 5. Simulation capacitances.

As it can be observed in Fig 5, the calculated capacitance considering the edge-effect of the conductors and the volume that surrounds these, are slightly bigger than the calculated capacitance taking in account the simplifications of Y. Xiang [12] (see Fig 2).

Thanks to the performance that offer the actual computers, it is possible to calculate the capacitance of any conductor system and to obtain polynomical equations that serve as calculation tool for the industrial making of complex systems, which are the most frequent cases in the field of the hybrid microelectronics.

\section{References}

[1] P.S.A.Evans, B.J.Ramsey, P.M.Harrey, D.J.Harrison. Printed analogue filter structures, Electron. Lett. 35 (1999) 306-308. 
[2] Y. Ishikawa, K. Hirose, M. Asayama, Y. Yamato, and S. Kobayashi, Dependence of current interruption performance on the element patterns of etched fuses, in: Proceedings of 8th International Conference on Electric Fuses and their Applications, 10-12 September 2007, Clermont-Ferrand, France, (2007) 51-56.

[3] J.Schreurs, J.Johnson, I.McNab. Characterization of Thick Films Formed on Slip Rings During High Current Density Operation, IEEE Trans. Components. Hvbrids. Manuf. Technol. 4 (1981) 30-35.

[4] R.Bansevicius, J.A.Virbalis. Two-dimensional Braille readers based on electrorheological fluid valves controlled by electric field, Mechatronics 17 (2007) 570-577.

[5] J.A.Given, C.O.Hwang, M.Mascagni. First- and lastpassage Monte Carlo algorithms for the charge density distribution on a conducting surface, Phys. Rev. E. 66 (2002) 056704-056708.

[6] C.O.Hwang, T.Won. Edge charge singularity of conductors, J. Korean. Phys. Soc. 45 (2004) S551-S553.

[7] C.O.Hwang, T.Won. Last-passage algorithms for corner charge singularity of conductors, J. Korean. Phys. Soc. 47 (2005) S464-S466.
[8] C.O.Hwang, J.A.Given. Last-passage Monte Carlo algorithm for mutual capacitance, Phys. Rev. E. 74 (2006) 027701-027703.

[9] Release 9.0 Documentation for ANSYS, (C) 2005 SAS IP, Inc.

[10] Y.Xiang. The electrostatic capacitance of an inclined plate capacitor, J. Electrostat. 64 (2006) 29-34.

[11] Y.Xiang. Non-linear oscillation of the fluid in a plate capacitor, Commun. Nonlinear. Sci. Numer. Sim. 12 (2007) 652-662.

[12] Y.Xiang. Further study on electrostatic capacitance of an inclined plate capacitor, J. Electrostat. 66 (2008) 366-368.

[13] R.Bansevicius, J.A.Virbalis. Distribution of electric field in the round hole of plane capacitor, J. Electrostat. 64 (2006) 226-233.

[14] J.L.Manglano de Mas. Lecciones de Física, Artes Gráficas Soler S.A., Valencia, 1995.

[15] J.D'Errico, Polyfitn, Personal Communication 